\title{
Effect of Pneumococcal Conjugate Vaccine on the Natural Antibodies and Antibody Responses Against Protein Antigens From Streptococcus pneumoniae, Haemophilus influenzae and Moraxella catarrhalis in Children With Community-acquired Pneumonia
}

\author{
Dafne C. Andrade, MD, * Igor C. Borges, MD, * Peter V. Adrian, PhD, \& Andreas Meinke, PhD, 9

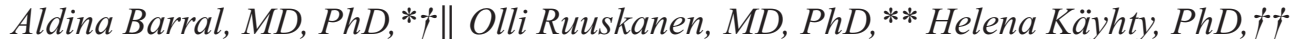 \\ and Cristiana M. Nascimento-Carvalho, MD, PhD*t
}

\begin{abstract}
Background: Streptococcus pneumoniae, Haemophilus influenzae and Moraxella catarrhalis are common causative agents of respiratory infections. Pneumococcal conjugate vaccines have been introduced recently, but their effect on the natural immunity against protein antigens from these pathogens has not been elucidated.

Methods: This was an age-matched observational controlled study that evaluated the influence of 10 -valent pneumococcal conjugate vaccines on the levels of antibodies and frequencies of antibody responses against proteins from S. pneumoniae, $H$. influenzae and M. catarrhalis in serum samples of children with community-acquired pneumonia. Eight pneumococcal proteins (pneumolysin, choline-binding protein A, pneumococcal surface protein A families 1 and 2, pneumococcal choline-binding protein $\mathrm{A}$, pneumococcal histidine triad protein $\mathrm{D}$, serine/threonine protein kinase, protein required for cell wall separation of group B streptococcus), 3 proteins from $H$. influenzae (including protein D) and 5 M. catarrhalis proteins were investigated.

Results: The study group comprised 38 vaccinated children and 114 agematched controls (median age: 14.5 vs. 14.6 months, respectively; $P=0.997$ ), all with community-acquired pneumonia. There was no difference on clinical baseline characteristics between vaccinated and unvaccinated children. Vaccinated children had significantly lower levels of antibodies against 4 of the studied pneumococcal antigens $(P=0.048$ for Ply, $P=0.018$ for pneumococcal surface protein A, $P=0.001$ for StkP and $P=0.028$ for PcsB) and higher levels of antibodies against $M$. catarrhalis $(P=0.015)$. Nevertheless, the vaccination status did not significantly affect the rates of antibody responses against $S$. pneumoniae, $H$. influenzae and M. catarrhalis.

Conclusions: In spite of the differences that have been found on the level of natural antibodies, no effect from pneumococcal vaccination was observed
\end{abstract}

Accepted for publication August 11, 2015

From the *Postgraduate Program in Health Sciences, $\uparrow$ Postgraduate Program in Human Pathology, \$Department of Paediatrics, Federal University of Bahia School of Medicine, Salvador Bahia, Brazil; §DST/NRF Vaccine Preventable Diseases, Respiratory and Meningeal Pathogens Research Unit, University of the Witwatersrand, Johannesburg, South Africa; qValneva Austria $\mathrm{GmbH}$, Campus Vienna Biocenter 3, Vienna, Austria; ICentro de Pesquisa Gonçalo Moniz, Fundação Oswaldo Cruz (FIOCRUZ), Salvador, Bahia, Brazil; **Department of Paediatrics, Turku University and University Hospital, Turku, Finland; $\dagger \dagger$ National Institute for Health and Welfare, Helsinki, Finland.

This study was supported by Bahia State Agency for Research Funding (FAPESB), Brazil; Brazilian Council for Scientific and Technological Development (CNPq), Brazil. Andreas Meinke is an employee at Valneva Austria $\mathrm{GmbH}$. The authors have no conflicts of interest to disclose.

Address for correspondence: Dafne C. Andrade, MD, Federal University of Bahia School of Medicine, Praça XV de Novembro, s/n-Largo do Terreiro de Jesus, Salvador, Bahia, Brazil, CEP 40025-010. E-mail: andradedafne@ yahoo.com.br.

Copyright (C) 2016 Wolters Kluwer Health, Inc. All rights reserved.

ISSN: 0891-3668/16/3506-0683

DOI: $10.1097 /$ INF.0000000000001126 on the rate of immune responses associated with community-acquired pneumonia against protein antigens from S. pneumoniae, H. influenzae and M. catarrhalis.

Key Words: humoral antibody response, immune factors, lower respiratory tract infection, polysaccharides, serological tests

(Pediatr Infect Dis J 2016;35:683-689)

$S_{e l l}^{\text {tres }}$ reptococcus pneumoniae, Haemophilus influenzae and Moraxella catarrhalis are common bacteria associated with acute respiratory infections in childhood, such as community-acquired pneumonia $(\mathrm{CAP})^{1}$ and acute otitis media. ${ }^{2}$ Furthermore, these bacteria also frequently colonize the nasopharyx, ${ }^{3-5}$ which might be considered the initial step of their pathogenesis. ${ }^{6}$ As a preventive strategy to control the burden of disease by these agents, polysaccharide-based conjugate vaccines have been introduced against $S$. pneumoniae ${ }^{7}$ and $H$. influenzae type b.

Pneumococcal conjugate vaccines (PCV) are being increasingly implemented worldwide. As a consequence, the incidence of disease and carriage of vaccine-covered serotypes has decreased. . $^{2,5}$ Nevertheless, an increase in colonization by nonvaccine serotypes has been identified, ${ }^{2}$ which may represent a limitation for the use of polysaccharide-based vaccines. As a result, new protein antigens have been reported as putative vaccine candidates due to their specificity and high degree of conservation among the strains of each bacterium. ${ }^{3-5}$

Few studies have addressed the development of natural antibodies and the frequency of antibody responses against protein antigens from $S$. pneumoniae, $H$. influenzae and $M$. catarrhalis. ${ }^{9-13}$ In addition, most of these studies were conducted before the introduction of the current polysaccharide-based vaccines (PCV and $H$. influenzae type b vaccine), which does not represent the general pediatric population anymore. We aimed to evaluate differences in the levels of natural antibodies and in the frequencies of antibody responses associated with CAP against protein antigens from $S$. pneumoniae, $H$. influenzae and $M$. catarrhalis in children who had been vaccinated or left unvaccinated with 10-valent PCV (PCV10).

\section{MATERIALS AND METHODS}

\section{Study Design and Participants}

This was an age-matched observational controlled study exploring the effects of vaccination with PCV10 on the levels of natural antibodies and frequency of antibody responses against protein antigens from S. pneumoniae, H. influenzae and M. catarrhalis. This study was part of the PNEUMOPAC-Efficacy trial ("Clinical 
Efficacy of Amoxicillin Given Twice or Three Times a Day Among Children With Non-severe Pneumonia" PNEUMOPAC-Efficacy trial (ClinicalTrials.gov NCT01200706, registered as "Clinical Efficacy of Amoxicillin Given Twice or Three Times a Day Among Children With Non-severe Pneumonia") ${ }^{14}$ in which 820 children aged 2-59 months old with nonsevere CAP were recruited in the Emergency Department of the Federal University of Bahia hospital in Salvador, Northeast Brazil, from November 2006 to May 2011. Inclusion criteria were report of respiratory complaints, detection of lower respiratory findings on physical examination and the presence of pulmonary infiltrate/consolidation on the chest radiograph taken on admission and read by the attending pediatrician. Exclusion criteria from this trial comprised the presence of chest indrawing or danger signs and underlying chronic diseases or immunodeficiency. Legal guardians from the included patients provided a written informed consent upon enrolment. Blood samples were collected at admission and 2-4 weeks later. After sample collection, they were immediately processed and stored at $-20^{\circ} \mathrm{C}$ until the moment of analysis. Sample collection was performed regardless of the completion of the vaccination schedule.

The use of PCV10 (Synflorix, GlaxoSmithKline Biologicals, Rixensart, Belgium) was universally introduced in Salvador, Brazil, on July 2010 for children aged $<2$ years. ${ }^{15}$ The scheduled vaccination regimen on the first year of implementation of this immunization program varied according to the age of the child. Children aged between 2 and 6 months received 3 doses of vaccine on the first year of life with a 2-month interval, with one booster dose at 12 months of age. Children aged 7-11 months who had not been previously vaccinated received 2 doses of PCV10, with a 2-month interval, along with a booster dose between 12 and 15 months of age. Finally, children aged 12-24 months only received one dose of PCV10, with no booster dose of the vaccine. ${ }^{15}$ Every child included in the PNEUMOPAC-efficacy trial who could have received PCV10 was identified based on the age and date of enrollment, comprising a group of 104 children. Each of these children had the vaccine card checked personally by one of the researchers (I.C.B.) after the trial was completed. Any dose of this vaccine given to those children was recorded along with the date of administration in a logbook. From these data, the number of doses of PCV10 and time interval between the first and last doses of PCV10 and the collection of the first serum sample were calculated.

In this observational controlled study, the studied cases were represented by vaccinated children from the PNEUMOPAC-Efficacy trial, and the controls were age-matched unvaccinated children from the same trial. Age matching was performed as a method to control for the potential effect of age on the levels of antibodies against the studied antigens. The primary criterium for the selection of controls was the age of unvaccinated children, which should be the closest ones to the age of the vaccinated cases. The case:control relation was 1:3. Only if more than 3 suitable controls had the same age difference to the vaccinated case, the entry order on the PNEUMOPAC-Efficacy trial was used to select the children who were enrolled first as controls.

Patients with severe malnutrition, defined as $\mathrm{Z}$ score for weight-for-age under $-3.00,{ }^{16}$ were excluded from this study. Nutritional evaluation was performed using "Anthro" software.

The PNEUMOPAC-Efficacy trial was approved by the Ethics Committee of the Federal University of Bahia and was conducted in accordance with the principles of the Declaration of Helsinki.

\section{Laboratory Procedures}

The concentration of antibodies against protein antigens from $S$. pneumoniae, $H$. influenzae and $M$. catarrhalis was determined with a multiplexed bead-based serological assay with Luminex
xMAP technology. ${ }^{17}$ The test included 8 recombinant proteins from $S$. pneumoniae: pneumolysin, choline-binding protein A, pneumococcal surface protein A families 1 and 2 (PspA 1 and 2), pneumococcal choline-binding protein $\mathrm{A}$, pneumococcal histidine triad protein $\mathrm{D}$, serine/threonine protein kinase (StkP-C, SP1732-3, a C-terminal fragment of StkP) and protein required for cell wall separation of group B streptococcus (PcsB-N, SP2216-1, a N-terminal fragment of PcsB), 3 recombinant proteins from $H$. influenzae (NTHi Protein D-the carrier protein in PCV10, heme/hemopexin utilization protein A [NTHi0371-1], and outer membrane antigenic lipoprotein B [NTHi0830]) and 5 recombinant proteins from $M$. catarrhalis (OMP CD, Msp22 [MC_RH4_2506], extracellular solute-binding protein [MC_RH4_1701], probable membrane-bound lytic murein transglycosylase D [MC_RH4_3729-1] and Methionine-R-sulfoxide reductase [MC_RH4_4730]). Using these antigens, 9 bead sets were produced using the following combination: pneumolysin, cholinebinding protein A, pneumococcal choline-binding protein A, pneumococcal histidine triad protein D, StkP-C and PcsB-N were conjugated in one bead region each; PspA 1 and PspA 2 were conjugated in the same bead region; and all $H$. influenzae and all $M$. catarrhalis proteins were conjugated in one bead region per bacterium.

For each antigen, the mean fluorescence intensity (MFI) values were obtained as an indirect measure of the immunoglobulin $\mathrm{G}$ concentration. Samples were assayed in duplicate and the results averaged. Acute and convalescent samples were always tested on the same plate. Furthermore, positive and negative controls were analyzed on each plate to ensure good batch-to-batch consistency. ${ }^{17}$ All samples were analyzed at 1:400 and 1:1600 dilutions, and when necessary, further dilutions were performed.

The levels of natural antibodies were defined as the MFI readings for each studied antigen measured on the first serum sample. The presence of an antibody response was defined as an increase on the antibody levels $\geq 2$-fold between the first and second serum samples. ${ }^{18}$ All the samples were tested from October 2012 to January 2013

\section{Statistical Analysis}

Categorical variables were compared using $\chi^{2}$ or Fisher exact test as appropriate and continuous variables were evaluated using Mann-Whitney $U$ test as they presented a nonparametric distribution. Correlation between 2 continuous variables was evaluated with Spearman correlation. Kruskal-Wallis test was applied to evaluate the association of a categorical variable with more than 2 levels and a continuous variable. Logistic regression was performed to evaluate the effect of continuous or categorical variables on a dichotomized variable, and linear regression was used to evaluate the effect of continuous or categorical variables on a continuous variable. The statistical tests were 2-tailed, with a significance level of 0.05. The software SPSS (version 9.0) was used for the analyses.

\section{RESULTS}

\section{Study Group}

Out of the 820 patients from PNEUMOPAC-Efficacy study, this study included 38 vaccinated patients and 114 agematched controls. Pairing between vaccinated and unvaccinated children according to age at admission was successful, and the maximum age difference between a case and its respective control was 10 days. The included unvaccinated children had similar demographic and serological characteristics when compared with the PNEUMOPAC cohort, whose data have been previously published. ${ }^{19}$ The comparison of the baseline clinical features for vaccinated and unvaccinated children demonstrated no significant difference between the study subgroups, as shown in Table 1 . 
TABLE 1. Comparison of Baseline Characteristics for Vaccinated (Cases) and Unvaccinated (Controls) Children

\begin{tabular}{lccc}
\hline \hline & \multicolumn{2}{c}{ PCV10 Vaccination } \\
\cline { 2 - 3 } & Vaccinated & Unvaccinated \\
& $(\mathrm{n}=38)$ & $(\mathrm{n}=114)$ & $P$ \\
\hline Sex (male) & $24(63.2 \%)$ & $65(57 \%)$ & 0.506 \\
Age (months) & $14.5(9.8-18.8)$ & $14.6(10-19)$ & 0.997 \\
Length of disease (days)* & $5(4-7.25)$ & $6(3-10)$ & 0.272 \\
Interval between samples & $17.5(16-20.25)$ & $19(16-21)$ & 0.590 \\
$\quad$ & & \\
Fever & $33(86.8 \%)$ & $103(90.4 \%)$ & 0.549 \\
Duration of fever (days)* & $3(2-5)$ & $4(2-5)$ & 0.324 \\
Cough & $35(92.1 \%)$ & $111(98.2 \%)$ & 0.102 \\
Duration of cough (days)* & $5(3-8)$ & $6(4-10)$ & 0.069 \\
Difficulty breathing & $25(65.8 \%)$ & $83(73.5 \%)$ & 0.365 \\
Duration of difficulty & $3(1.5-5)$ & $3(1-5)$ & 0.739 \\
$\quad$ breathing (days)* & & & \\
Wheezing & $12(31.6 \%)$ & $34(29.8 \%)$ & 0.838 \\
Duration of wheezing (days)* & $3(2-4)$ & $3(1-5)$ & 0.849 \\
Vomit & $16(42.1 \%)$ & $54(47.4 \%)$ & 0.573 \\
Duration of vomiting (days)* & $1(1-3)$ & $2(1-3)$ & 0.259 \\
\hline
\end{tabular}

*Continuous variables presented as median (25th-75th percentile).

On the date of the first serum sample collection, most vaccinated children (cases) had completed the appropriate vaccination schedule. Figure 1 shows the flowchart of the included and excluded cases of this study, along with the vaccination status and the number of doses of PCV administered to each vaccinated child included herein.

\section{Differences in the Levels of Natural Antibodies in Vaccinated and Unvaccinated Children}

The comparison of the MFI values, expressing the levels of natural antibodies against protein antigens from S. pneumoniae, $H$. influenzae and M. catarrhalis, between age-matched vaccinated and unvaccinated children with CAP using the 1:1600 dilution factor is shown in Table 2. Overall, vaccinated children presented lower levels of antibodies against most of the tested pneumococcal antigens and higher levels of antibodies against $M$. catarrhalis. No significant difference was seen for $H$. influenzae proteins. Similar results were found when data from the 1:400 dilution was used (data not shown).

\section{Antibody Responses Associated With CAP Against Protein Antigens From S. pneumoniae, H. influenzae and $M$. catarrhalis in Vaccinated and Unvaccinated Children}

The comparison of the frequency of responders for each studied antigen in age-matched vaccinated and unvaccinated children is shown in Table 3. No significant differences were found in the frequency of antibody responses between vaccinated and unvaccinated children. Overall, 7 patients presented decreases $\leq 0.5$ in the levels of antibodies against any protein from $S$. pneumoniae (vaccinated vs. unvaccinated: $2[5.3 \%]$ vs. $5[4.4 \%] ; P=1$ ). None of the patients presented decreases in the antibody levels against H. influenzae and M. catarrhalis.

\section{Effect of the Number of Doses of PCV10 and the Time Interval From the First/Last Dose of Vaccine on the Levels of Natural Antibodies and Frequency of Antibody Responses in Vaccinated Children}

There was no effect of number of administered doses of PCV10 on the levels of natural antibodies against protein antigens at either 1:400 or 1:1600 dilution using Kruskal-Wallis test (data not shown). No effect of time interval between the first or last dose of PCV10 and the collection of the first serum sample on the levels of natural antibodies against protein antigens was found for S. pneumoniae and M. catarrhalis, as shown in Table 4. There was a positive association between the time interval from the first dose of PCV10 to the collection of the first serum sample and the level of natural antibodies against $H$. influenzae. However, when a linear regression was performed adjusting the aforementioned association by the age of the child upon enrollment, no effect of the time interval between the first dose of PCV10 and the collection of the first serum sample on the levels of antibodies against $H$. influenzae was detected $(P=0.697)$

Neither the number of doses of PCV10 nor the time interval between the administration of the first/last dose of PCV10 and the collection of the first serum sample influenced the frequency of antibody responses against the studied protein antigens when evaluated using logistic regression (data not shown).

\section{DISCUSSION}

Our case-control study demonstrated that there is a significant difference in the levels of natural antibodies against protein antigens from $S$. pneumoniae and $M$. catarrhalis between agematched children who were vaccinated with PCV10 or did not receive this vaccine. Nevertheless, there was no difference between vaccinated and unvaccinated in the frequency of antibody responders against the studied antigens upon contraction of CAP.

Of note, vaccinated children had significantly lower MFI values to 4 of the evaluated pneumococcal antigens (pneumolysin, PspA, StkP and PcsB). Also, the antibody levels against cholinebinding protein $\mathrm{A}$, pneumococcal choline-binding protein $\mathrm{A}$ and pneumococcal histidine triad protein D were lower in vaccinated children compared with unvaccinated controls, but this did not reach statistical significance. Similarly, in the study by Prevaes et al, ${ }^{9}$ there was also a trend toward lower MFI readings in vaccinated children for the antigens Nan, Pilus A, PspA and PsaA in 24-month-old children. Conversely, Ditse et $\mathrm{al}^{13}$ found no difference on the levels of antibodies against protein antigens between children vaccinated with PCV7 or unvaccinated. Nevertheless, the study population from Ditse et $\mathrm{al}^{13}$ was significantly older than the one herein, which might compromise the comparison of the effect of pneumococcal vaccination due to the long-term decrease in effectiveness of PCVs after primary vaccination. ${ }^{20}$ Accordingly, no effect of vaccination on the prevalence of overall or vaccine-serotype colonization by S. pneumoniae was found in the aforementioned study. ${ }^{13,20}$ In addition, this study is the first evaluating the effect of PCV10 on the level of natural anti-protein antibodies in vaccinated and unvaccinated children. Consequently, it is possible that the greater coverage provided by PCV10 when compared with the PCVs used in previous studies might have influenced the difference in the natural levels of antibodies between vaccinated and unvaccinated children.

The lower MFI values found for vaccinated children might have been caused by the effects of PCV10 on the rate of nasopharyngeal colonization. For instance, the use of PCV10 may have reduced the overall rate of pneumococcal carriage for the studied children as it has already been reported in previous vaccine trials with other types of $\mathrm{PCV}^{21,22}$ In this setting, by decreasing the carriage rates of $S$. pneumoniae, vaccinated children would not be exposed to numerous pneumococcal protein antigens. ${ }^{23}$ This, in turn, might have reduced the production of antipneumococcal antibodies in this subgroup of children. Indeed, it has already been demonstrated that children frequently colonized with $S$. pneumoniae present higher levels of antiprotein antibodies compared with those in which colonization was not found. ${ }^{24}$ 


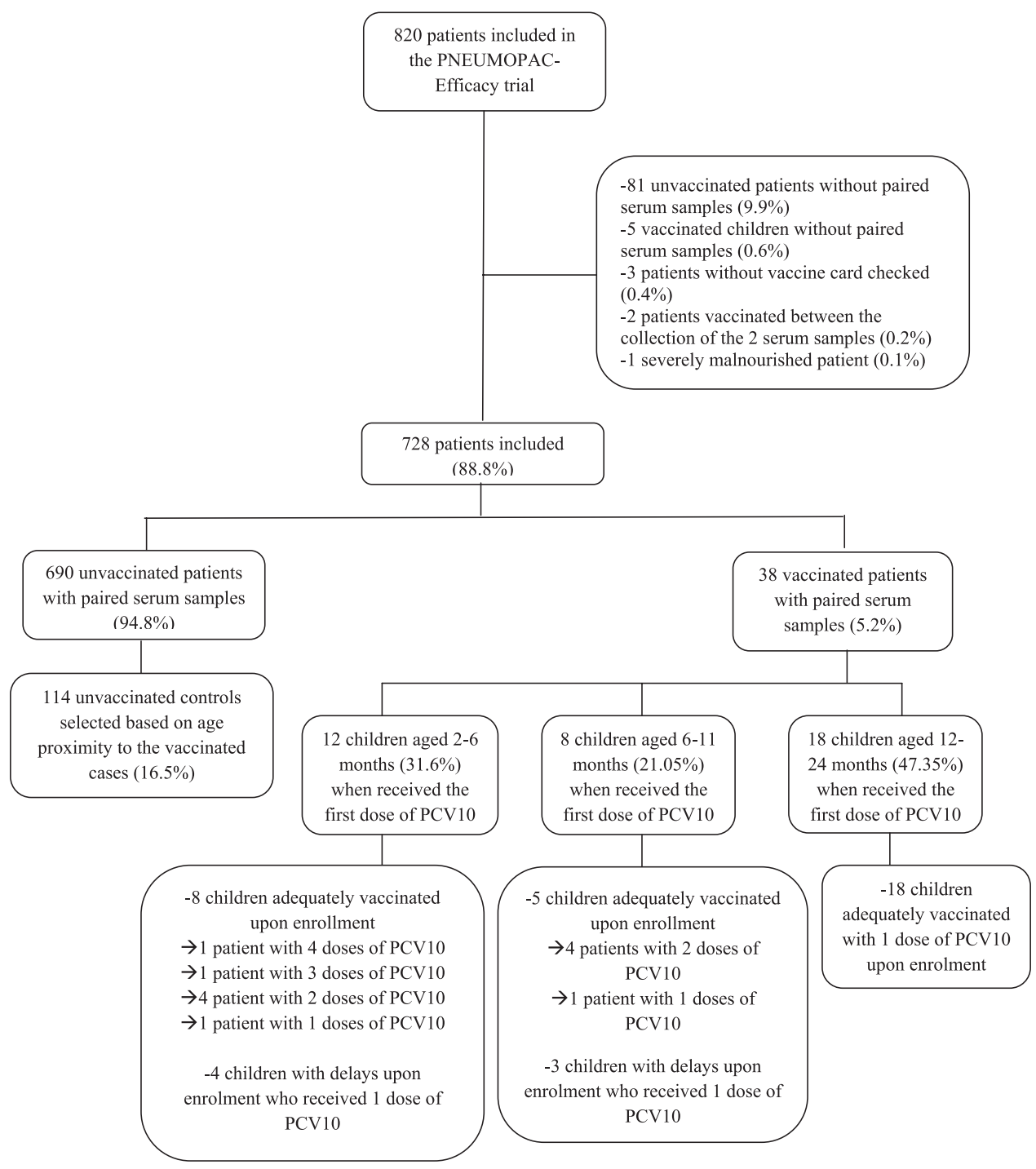

FIGURE 1. Flow chart of the included and excluded cases of this study and description of the vaccination status and the number of doses of PCV administered to each vaccinated child.

TABLE 2. Comparison of the Antibody Levels of the First Serum Sample Taken Upon Enrollment With a Dilution Factor of 1:1600 Between Vaccinated (Cases) and Unvaccinated (Controls) Children

\begin{tabular}{lccc}
\hline \hline & \multicolumn{2}{c}{ MFI Values (Median [25th-75th percentile]) } & \\
\cline { 2 - 3 } \multicolumn{1}{c}{ Protein } & $\begin{array}{c}\text { Vaccinated } \\
(\mathrm{n}=38)\end{array}$ & $\begin{array}{c}\text { Unvaccinated } \\
(\mathrm{n}=114)\end{array}$ & \\
\hline Ply & $57(35.5-113.75)$ & $74(43.5-189)$ & 0.048 \\
CbpA & $1224(124-3707)$ & $2064(242.75-5832.75)$ & 0.171 \\
PspA* & $112(53.5-266)$ & $191(84.25-594.75)$ & 0.018 \\
PcpA & $363(38.5-1010.5)$ & $637.5(69.5-1380.75)$ & 0.074 \\
PhtD & $435(80-1034)$ & $735.5(104.75-2350)$ & 0.089 \\
StkP & $101(30-139.75)$ & $162(47.75-594)$ & 0.001 \\
PcsB & $443(64-1677.25)$ & $1212.5(104.5-3380.5)$ & 0.028 \\
Haemophilus influenzae & $130(56.75-207.75)$ & $110.5(79.5-196.5)$ & 0.670 \\
Moraxella catarrhalis & $149.5(104-303.75)$ & $111(76-188.25)$ & 0.015 \\
\hline
\end{tabular}

*PspA 1 and 2 were conjugated on the same bead set.

CbpA indicates choline-binding protein A; PcpA, pneumococcal choline-binding protein A; PcsB, protein required for cell wall separation of group B streptococcus; PhtD, pneumococcal histidine triad protein D; Ply, pneumolysin; StkP, serine/threonine protein kinase. 
TABLE 3. Comparison of the Frequency of Antibody Response Detection Between Vaccinated (Cases) and Unvaccinated (Controls) Children With CAP

\begin{tabular}{|c|c|c|c|}
\hline \multirow[b]{2}{*}{ Protein } & \multicolumn{2}{|c|}{$\begin{array}{l}\text { Frequency of Antibody } \\
\text { Increase (n [\%]) }\end{array}$} & \multirow[b]{2}{*}{$P$} \\
\hline & $\begin{array}{l}\text { Vaccinated } \\
\qquad(\mathrm{N}=38)\end{array}$ & $\begin{array}{l}\text { Unvaccinated } \\
\qquad(\mathrm{N}=114)\end{array}$ & \\
\hline Ply & $0(0 \%)$ & $3(2.6 \%)$ & 0.573 \\
\hline CbpA & $2(5.3 \%)$ & $3(2.6 \%)$ & 0.599 \\
\hline PspA* & $0(0 \%)$ & $2(1.8 \%)$ & 1 \\
\hline PcpA & $1(2.6 \%)$ & $9(7.9 \%)$ & 0.453 \\
\hline PhtD & $0(0 \%)$ & $6(5.3 \%)$ & 0.337 \\
\hline StkP & $2(5.3 \%)$ & $6(5.3 \%)$ & 1 \\
\hline $\mathrm{PcsB}$ & $1(2.6 \%)$ & $6(5.3 \%)$ & 0.681 \\
\hline Streptococcus pneumoniae $\dagger$ & $4(10.5 \%)$ & $17(14.9 \%)$ & 0.497 \\
\hline Haemophilus influenzae & $4(10.5 \%)$ & $12(10.5 \%)$ & 1 \\
\hline Moraxella catarrhalis & $4(10.5 \%)$ & $4(3.5 \%)$ & 0.108 \\
\hline \multicolumn{4}{|c|}{ Responses to multiple pathogens } \\
\hline $\begin{array}{l}\text { S. pneumoniae and } \\
\text { H. influenzae }\end{array}$ & $2(5.3 \%)$ & $1(0.9 \%)$ & 0.154 \\
\hline $\begin{array}{l}\text { S. pneumoniae and } \\
\text { M. catarrhalis }\end{array}$ & $0(0 \%)$ & $2(1.8 \%)$ & 1 \\
\hline $\begin{array}{l}\text { H. influenzae and } \\
\text { M. catarrhalis }\end{array}$ & $0(0 \%)$ & $0(0 \%)$ & - \\
\hline $\begin{array}{l}\text { S. pneumoniae, } H \text {. influenzae } \\
\text { and } M \text {. catarrhalis }\end{array}$ & $0(0 \%)$ & $0(0 \%)$ & - \\
\hline Overall detection rate $\ddagger$ & $10(26.3 \%)$ & $29(25.4 \%)$ & 1 \\
\hline
\end{tabular}

*PspA 1 and 2 were conjugated on the same bead set.

$\dagger$ Frequency of antibody response detection to at least one pneumococcal protein. $\doteqdot$ Detection of responses against any of the studied pathogens.

CbpA indicates choline-binding protein A; PcpA, pneumococcal choline-binding protein A; PcsB, protein required for cell wall separation of group B streptococcus; PhtD, pneumococcal histidine triad protein D; Ply, pneumolysin; StkP, serine/threonine protein kinase.

TABLE 4. Effect of the Time Interval Between the Date of Administration of the First and/or Last Dose of PCV10 and the Date of Collection of the First Serum Sample on the Levels of Natural Antibodies Against Protein Antigens

\begin{tabular}{|c|c|c|c|c|}
\hline \multirow{2}{*}{$\begin{array}{l}\text { Antibody } \\
\text { Concentration in } \\
\text { the First Serum } \\
\text { Sample Against }\end{array}$} & \multicolumn{2}{|c|}{$\begin{array}{l}\text { Time Interval Between } \\
\text { the First Dose of PCV } \\
\text { and Collection of } \\
\text { the First Sample }\end{array}$} & \multicolumn{2}{|c|}{$\begin{array}{c}\text { Time Interval Between } \\
\text { the Last Dose of PCV } \\
\text { and Collection of } \\
\text { the First Sample }\end{array}$} \\
\hline & $r^{*}$ & $P^{*}$ & $r^{*}$ & $P^{*}$ \\
\hline Ply & 0.041 & 0.805 & 0.122 & 0.467 \\
\hline CbpA & -0.158 & 0.344 & -0.044 & 0.793 \\
\hline PspA $\dagger$ & -0.099 & 0.555 & 0.067 & 0.688 \\
\hline PcpA & 0.036 & 0.829 & 0.017 & 0.921 \\
\hline PhtD & -0.085 & 0.612 & -0.038 & 0.819 \\
\hline StkP & -0.174 & 0.297 & 0.036 & 0.829 \\
\hline PcsB & -0.112 & 0.505 & -0.066 & 0.695 \\
\hline $\begin{array}{l}\text { Haemophilus } \\
\text { influenzae }\end{array}$ & 0.384 & 0.017 & 0.151 & 0.367 \\
\hline $\begin{array}{l}\text { Moraxella } \\
\quad \text { catarrhalis }\end{array}$ & 0.124 & 0.460 & 0.243 & 0.141 \\
\hline
\end{tabular}

*Similar results were found when using a 1:400 dilution factor (data not shown).

$†$ PspA 1 and 2 were conjugated on the same bead set.

CbpA indicates choline-binding protein A; PcpA, pneumococcal choline-binding protein A; PcsB, protein required for cell wall separation of group B streptococcus; PhtD, pneumococcal histidine triad protein D; Ply, pneumolysin; $r$, correlation coefficient; $\mathrm{StkP}$, serine/threonine protein kinase.

Despite the differences of the antibody levels, no statistically significant difference between vaccinated and unvaccinated children was found regarding the frequency of antibody responders against the studied pneumococcal proteins upon contraction of CAP. A possible explanation for this finding is that in vaccinated children, disease was caused by nonvaccine serotypes. Increases in the rate of invasive pneumococcal disease caused by nonvaccine serotypes have been reported after the introduction of PCVs. ${ }^{25}$ The antigens used in this study are, however, highly conserved and almost universally present in all strains of $S$. pneumoniae. ${ }^{23,26-31}$ Therefore, the antibody response against them should not be substantially affected by the serotype of pneumococcus causing CAP. In addition, high levels of several antiprotein pneumococcal antibodies reduce adherence of the pneumococcus to human lung epithelial cells ${ }^{32}$ and have been associated with a reduced frequency of acute respiratory infections in children. ${ }^{10,13}$ Therefore, although children vaccinated with PCV10 were protected against disease caused by the vaccine-covered serotypes, they lacked the protective effect of high levels of antiprotein antibodies, rendering them sensitive to nonvaccine serotypes. This fact, in turn, might also have contributed to the similar frequency of antibody response found for vaccinated and unvaccinated children. However, it is important to recall that this study evaluated children with nonsevere pneumonia treated as outpatients, in whom viruses have been demonstrated to be the major cause of CAP instead of bacteria. ${ }^{33,34}$ Therefore, a lower impact of pneumococcal vaccination is expected in this group of patients.

No difference was found either for the natural antibody levels or the frequency of antibody responders against $H$. influenzae associated with CAP between vaccinated and unvaccinated children. On the other hand, vaccinated children presented higher levels of antibodies against $M$. catarrhalis when compared with unvaccinated children. One possible explanation is that vaccinated children might have had a greater carriage rate for this bacterium as colonization by the common vaccine serotypes of $S$. pneumoniae was prevented and possibly allowed a higher rate of colonization by $M$. catarrhalis. Accordingly, changes in the rate of nasopharyngeal colonization by $M$. catarrhalis have already been reported in certain circumstances known to affect the local flora, such as day-care attendance. ${ }^{35}$ However, most studies evaluating the rates of colonization by $M$. catarrhalis after pneumococcal vaccination found no difference between vaccinated and unvaccinated children. ${ }^{36,37}$

The limitations of our study must be acknowledged. First, the group of children who received PCV10 was small and heterogeneous regarding the completion of the vaccination scheme and the number of PCV10 doses that were administered. Nevertheless, we found no effect for the number of doses of PCV10 or for the time interval between the first/last dose of vaccine and first serum sample collection on either the levels of natural antibodies or the frequency of antibody responses in this group of children. This, in turn, might suggest that although the group of vaccinated children was indeed heterogeneous, the differences of the vaccination schemes did not significantly affect the outcome variables from this study. Also, the groups of vaccinated children and their respective unvaccinated controls were very similar with regard to age and other clinical features, so that the differences found herein cannot be explained by these factors. It is important to recall that this was an exploratory study, and our findings should be confirmed in studies with a larger and homogeneous sample regarding vaccination status. Second, we had no data from the colonization status from the evaluated children or definitive tests on the etiology of CAP. Therefore, the association between the findings from this study and putative changes in the rates of colonization or etiology of CAP are only theoretical. Finally, we had no data on the use of other vaccines which might have affected the rates of colonization/infection by the studied pathogens, such as the $H$. influenzae type $\mathrm{b}$ vaccine. Nevertheless, it has already been demonstrated that the coverage of the $H$. influenzae type $\mathrm{b}$ vaccine is high in the pediatric population in Brazil, so it probably did not affect the results from this study. ${ }^{38}$ 
In conclusion, this study demonstrated that children who were vaccinated with PCV10 had lower levels of natural antibodies against 4 protein antigens from $S$. pneumoniae when compared with unvaccinated children with similar age and clinical presentation. This could reflect lower $S$. pneumoniae carriage rates in the vaccinated children as a consequence of PCV10 vaccination. Despite the differences in the levels of natural antibodies, no difference was found in the rates of antibody responders associated with CAP against protein antigens from $S$. pneumoniae, $H$. influenzae and $M$. catarrhalis among vaccinated and unvaccinated children with CAP

\section{ACKNOWLEDGMENTS}

We thank Sanofi Pasteur (Lyon, France) for supplying PcpA and PhtD;Elaine Tuomanen at St. Judes Children's Research Hospital (Memphis, USA) for supplying Ply, CbpA, PspA1; Susan Hollingshead, David Briles and Pat Coan at University of Alabama (Birmingham, USA) for supplying PspA2; and Valneva Austria $\mathrm{GmbH}$ (Vienna, Austria) for supplying StkP-C, PcsB-N, NTHi Protein D, NTHi0371-1, NTHi0830, MC Omp CD, MC RH4 2506, MC_RH4_1701, MC_RH4_3729-1 and MC_RH4_4730. We also thank Nina Ekström, Hanna Laitinen, Camilla Virta and Leena Saarinen for their help in the laboratory.

\section{REFERENCES}

1. Rudan I, Boschi-Pinto C, Biloglav Z, et al. Epidemiology and etiology of childhood pneumonia. Bull World Health Organ. 2008;86:408-416.

2. Casey JR, Adlowitz DG, Pichichero ME. New patterns in the otopathogens causing acute otitis media six to eight years after introduction of pneumococcal conjugate vaccine. Pediatr Infect Dis J. 2010;29:304-309.

3. Poolman JT, Bakaletz L, Cripps A, et al. Developing a nontypeable Haemophilus influenzae (NTHi) vaccine. Vaccine. 2000;19(suppl 1):S108S115.

4. Murphy TF, Parameswaran GI. Moraxella catarrhalis, a human respiratory tract pathogen. Clin Infect Dis. 2009;49:124-131.

5. van der Poll T, Opal SM. Pathogenesis, treatment, and prevention of pneumococcal pneumonia. Lancet. 2009;374:1543-1556.

6. Bogaert D, De Groot R, Hermans PW. Streptococcus pneumoniae colonisation: the key to pneumococcal disease. Lancet Infect Dis. 2004;4:144-154.

7 Rodgers GL, Klugman KP. The future of pneumococcal disease prevention. Vaccine 2011; 29: 43-48.

8. Prymula R, Kriz P, Kaliskova E, et al. Effect of vaccination with pneumococcal capsular polysaccharides conjugated to Haemophilus influenzaederived protein $\mathrm{D}$ on nasopharyngeal carriage of Streptococcus pneumoniae and $H$. influenzae in children under 2 years of age. Vaccine. 2009;28:71-78.

9. Prevaes SM, van Wamel WJ, de Vogel CP, et al. Nasopharyngeal colonization elicits antibody responses to staphylococcal and pneumococcal proteins that are not associated with a reduced risk of subsequent carriage. Infect Immun. 2012;80:2186-2193.

10. Lebon A, Verkaik NJ, Labout JA, et al. Natural antibodies against several pneumococcal virulence proteins in children during the pre-pneumococcalvaccine era: the generation R study. Infect Immun. 2011;79:1680-1687.

11. Verhaegh SJ, Stol K, de Vogel CP, et al. Comparative analysis of the humoral immune response to Moraxella catarrhalis and Streptococcus pneumoniae surface antigens in children suffering from recurrent acute otitis media and chronic otitis media with effusion. Clin Vaccine Immunol. 2012;19:914-918.

12. Holmlund E, Quiambao B, Ollgren J, et al. Antibodies to pneumococcal proteins PhtD, CbpA, and LytC in Filipino pregnant women and their infants in relation to pneumococcal carriage. Clin Vaccine Immunol. 2009;16:916-923.

13. Ditse Z, Adrian PV, Kuwanda L, et al. Association of Streptococcus pneumoniae common protein antigen (CPA) antibodies and pneumococcal nasopharyngeal colonization in HIV-infected and HIV-uninfected African children. Vaccine. 2013;31:4421-4427.

14. Vilas-Boas AL, Fontoura MS, Xavier-Souza G, et al; PNEUMOPACEfficacy Study Group. Comparison of oral amoxicillin given thrice or twice daily to children between 2 and 59 months old with non-severe pneumonia: a randomized controlled trial. J Antimicrob Chemother. 2014;69: $1954-1959$
15. Secretaria de Saúde do Estado da Bahia. Introdução da vacina pneumocócica 10-valente (conjugada) no calendário básico de vacinação da criança. Secretaria de Saúde do Estado da Bahia, Salvador, Brasil [Department of Health of the State of Bahia]. Introduction of the 10-valent pneumococcal conjugate vaccine in the basic childhood immunization schedule. [Department of Health of the State of Bahia, Salvador, Brazil]. 2010; Available at: http://www.vigilanciaemsaude.ba.gov.br/sites/default/files/ Boletim Implantação da Pneumo 10.pdf. Accessed March 13, 2014

16 World Health Organization (WHO). Training Course on Child Growth Assessment. Geneva, Switzerland: World Health Organization. 2008; Available at: http://whqlibdoc.who.int/publications/2008/978924159507 0_A_eng.pdf. Accessed July 13, 2009

17. Andrade DC, Borges IC, Laitinen H, et al. A fluorescent multiplexed beadbased immunoassay (FMIA) for quantitation of IgG against Streptococcus pneumoniae, Haemophilus influenzae and Moraxella catarrhalis protein antigens. J Immunol Methods. 2014;405:130-143.

18. Nohynek H, Eskola J, Kleemola M, et al. Bacterial antibody assays in the diagnosis of acute lower respiratory tract infection in children. Pediatr Infect Dis J. 1995;14:478-484.

19. Borges IC, Andrade DC, Vilas-Boas AL, et al. Detection of antibody responses against Streptococcus pneumoniae, Haemophilus influenzae, and Moraxella catarrhalis proteins in children with community-acquired pneumonia: effects of combining pneumococcal antigens, pre-existing antibody levels, sampling interval, age, and duration of illness. Eur J Clin Microbiol Infect Dis. 2015;34:1551-1557.

20. Madhi SA, Adrian P, Kuwanda L, et al. Long-term effect of pneumococcal conjugate vaccine on nasopharyngeal colonization by Streptococcus pneumoniae-and associated interactions with Staphylococcus aureus and Haemophilus influenzae colonization-in HIV-Infected and HIV-uninfected children. J Infect Dis. 2007;196:1662-1666.

21. Spijkerman J, Prevaes SM, van Gils EJ, et al. Long-term effects of pneumococcal conjugate vaccine on nasopharyngeal carriage of $S$. pneumoniae, $S$ aureus, H. influenzae and M. catarrhalis. PLoS One. 2012;7:e39730.

22. van Gils EJ, Veenhoven RH, Hak E, et al. Effect of reduced-dose schedules with 7-valent pneumococcal conjugate vaccine on nasopharyngeal pneumococcal carriage in children: a randomized controlled trial. JAMA 2009;302:159-167.

23. Tai SS. Streptococcus pneumoniae protein vaccine candidates: properties, activities and animal studies. Crit Rev Microbiol. 2006;32:139-153.

24. Pichichero ME, Kaur R, Casey JR, et al. Antibody response to Streptococcus pneumoniae proteins $\mathrm{PhtD}$, LytB, PcpA, PhtE and Ply after nasopharyngeal colonization and acute otitis media in children. Hum Vaccin Immunother. 2012;8:799-805

25. Flasche S, Van Hoek AJ, Sheasby E, et al. Effect of pneumococcal conjugate vaccination on serotype-specific carriage and invasive disease in England: a cross-sectional study. PLoS Med. 2011;8:e1001017.

26. Brooks-Walter A, Briles DE, Hollingshead SK. The pspC gene of Streptococcus pneumoniae encodes a polymorphic protein, PspC, which elicits cross-reactive antibodies to PspA and provides immunity to pneumococcal bacteremia. Infect Immun. 1999;67:6533-6542.

27. Khan MN, Sharma SK, Filkins LM, et al. PcpA of Streptococcus pneumoniae mediates adherence to nasopharyngeal and lung epithelial cell and elicits functional antibodies in humans. Microbes Infect. 2012;14: 1102-1110.

28. Khan MN, Pichichero ME. Vaccine candidates PhtD and PhtE of Streptococcus pneumoniae are adhesins that elicit functional antibodies in humans. Vaccine. 2012;30:2900-2907.

29. Adamou JE, Heinrichs JH, Erwin AL, et al. Identification and characterization of a novel family of pneumococcal proteins that are protective against sepsis. Infect Immun. 2001;69:949-958.

30. Giefing C, Meinke AL, Hanner M, et al. Discovery of a novel class of highly conserved vaccine antigens using genomic scale antigenic fingerprinting of pneumococcus with human antibodies. J Exp Med. 2008;205:117-131.

31. Croney CM, Coats MT, Nahm MH, et al. PspA family distribution, unlike capsular serotype, remains unaltered following introduction of the heptavalent pneumococcal conjugate vaccine. Clin Vaccine Immunol. 2012;19:891-896.

32. Kaur R, Surendran N, Ochs M, et al. Human antibodies to PhtD, PcpA, and Ply reduce adherence to human lung epithelial cells and murine nasopharyngeal colonization by Streptococcus pneumoniae. Infect Immun 2014;82:5069-5075.

33. Bradley JS, Byington CL, Shah SS, et al. Executive summary: the management of community-acquired pneumonia in infants and children older than 3 months of age: clinical practice guidelines by the Pediatric Infectious 
Diseases Society and the Infectious Diseases Society of America. Clin Infect Dis. 2011;53:617-630.

34. Ruuskanen O, Lahti E, Jennings LC, et al. Viral pneumonia. Lancet. 2011;377:1264-1275.

35. Verhaegh SJ, Lebon A, Saarloos JA, et al. Determinants of Moraxella catarrhalis colonization in healthy Dutch children during the first 14 months of life. Clin Microbiol Infect. 2010;16:992-997.

36. van Gils EJ, Veenhoven RH, Rodenburg GD, et al. Effect of 7-valent pneumococcal conjugate vaccine on nasopharyngeal carriage with Haemophilus influenzae and Moraxella catarrhalis in a randomized controlled trial. Vaccine. 2011;29:7595-7598.

37. Dunne EM, Manning J, Russell FM, et al. Effect of pneumococcal vaccination on nasopharyngeal carriage of Streptococcus pneumoniae, Haemophilus influenzae, Moraxella catarrhalis, and Staphylococcus aureus in Fijian children. J Clin Microbiol. 2012;50:1034-1038.

38 Rede Interagencial de Informações para a Saúde. Cobertura Vacinal. [Interagency Network of Information for Health. Vaccine Coverage]. 2008; Available at: http://www3.saude.ba.gov.br/cgi/tabcgi.exe?tabnet/ripsa/f13/ f13.def. Accessed June 20, 2014. 